\title{
Evolution of Bearing Determination IN HF CURRENT MAPPING RADARS
}

\author{
By Donald E. Barrick and Belinda J. Lipa
}

\section{Much of the effort \\ in $\mathrm{HF}$ radar hardware \\ and software re- \\ search . . has been \\ in the development of \\ smaller, affordable}

antenna systems . . .
$\mathrm{N}$ tence operate at microwave frequencies because their wavelengths are small enough that compact antennas provide both good angular resolution and high sensitivity. By contrast, at high frequency (HF) very large antenna arrays are needed to achieve similar results using traditional signal processing techniques. (To form and scan a beam equal in width to that from a $2-\mathrm{m}$ microwave dish demands an $H F$ receive antenna that is $2-3 \mathrm{~km}$ in length.) Despite this physical drawback, only at HF is the first and second-order sea echo directly relatable to surface waves and winds and, through the Doppler relation, to surface currents (Crombie, 1955: Barrick, 1972). For example, at microwave frequencies Doppler shifts depend on many scattering properties of the surface in addition to the wave and current speeds.

At $\mathrm{HF}$ as well as at microwave, range to a radar cell can be obtained accurately from the echo's time delay: Doppler is likewise obtained from spectral analysis of the echo time series. The problem lies in the accurate determination of bearing. Much of the effort in HF radar hardware and software research over the past 20 years has been in the development of smaller. affordable antenna systems that maintain the bearing accuracy of the larger antenna arrays. Our paper summarizes the latest advance in this quest, describing a powerful new direction finding (DF) algorithm called MU1tiple SIgnal Classification (MUSIC), which is particularly well suited for application to HF radar sea echo. Its performance is demonstrated using simulation analyses.

\section{Direction Finding Bearing Estimation}

As old as radio itself, the simplest DF system is a loop antenna rotated until the incoming signal vanishes. Knowledge of this null direction and the angular response function of the antenna provides

Donald. E. Barrick and Belinda J. Lipa. CODAR Ocean Sensors, LTD., 1000 Fremont Ave., Los Altos. CA 94024. USA. information about the direction from which the radio signal is originating.

Use of DF for current mapping began in 1975 . The most compact realizations of DF techniques for this purpose have been the CODAR-type HF radars, which employ two crossed loops mounted around a whip (vertical monopole). An example of this antenna configuration is shown in Figure 1, along with stylized plots of the ideal angular amplitude patterns for each of the three elements. In addition to the theoretical (and achieved) shape of the antenna patterns, the accuracy of all DF algorithms also depend on the signal-to-noise $(\mathrm{S} / \mathrm{N})$ ratio of the measured backscatter.

Historically, current-mapping DF antennas and algorithms have taken several forms. Barrick et al. (1977) and Gurgel (1997) used antenna elements separated by short distances and relied on phasepath differences to extract bearing. We focus here on the most compact systems where the antenna elements are colocated. Their inherent amplitude and phase pattern differences have led to use of the following DF techniques:

1. The simplest algorithm, which takes the ratio of signals from the two loops (with sine and cosine patterns) and the whip (with omni-directional reception), and extracts bearing using the arctangent function. This closed-form algorithm is applied to each spectral bin constituting the Bragg peak. Although conceptually simple, this method is not robust in the presence of spatially complex current fields or when antenna patterns are distorted.

2. A least-squares algorithm that best-fits a model for each received Doppler frequency to measured cross spectra among the three antenna signals as demonstrated by Lipa and Barrick (1983). This method can handle both single- and dual-angle solutions ( 1 or 2 signals at the same frequency from different bearings) and provides limited statistical means for testing which solution fits the data better. Unlike closed-form solutions, the method can incorporate measured antenna patterns that may be distorted from the ideal patterns by nearby environmental obstacles (Barrick and 
Lipa, 1986). Used for 15 years, this method suffers from the following two defects: 1) it is numerically inefficient because it uses a two-dimensional grid search to find best solutions to the nonlinear problem; hence, it is not easily extended to more complex antennas and 2) the covariance matrix among antenna signals for sea echo is singular because they are correlated. This inefficiency restricts the use of objective hypothesis testing to select between single and dual-angle solutions.

3. The MUSIC algorithm as presented by Schmidt (1986). Using an eigenfunction analysis of signals received on $\mathrm{N}$ antennas, MUSIC can find up to N-1 signal bearings at the same frequency. It takes advantage of the covariance matrix singularity in extracting bearing.

\section{MUSIC Applied to HF Current Mapping}

We provide here a stepwise procedural outline for the application of MUSIC to the HF direction finding problem:

- Form a sample covariance matrix of the complex spectrally analyzed signals from the $\mathrm{N}$ antenna elements. (For the SeaSonde with $\mathrm{N}=3$, this is $3 \times 3$.) Each spectral bin corresponds to a known current radial velocity, which is the independent variable of the problem. In practice, several consecutive cross spectra are averaged to get the sample covariance matrix.

- Perform an eigenfunction analysis of the covariance matrix and order the eigenvectors from largest to smallest by eigenvalue. The biggest of these represents sea echo from one or more bearings at the given radial velocity, whereas the smaller eigenvectors are noise. By definition, all of the eigenvectors are mutually orthogonal.

- Create the signal-model vector from the known antenna patterns. For our idealized case, these are sine, cosine, and constant functions of angle. If the patterns are distorted, their measured responses are used instead. This becomes an $\mathrm{N}$-element vector.

- Determine candidate bearings using the fundamental principle behind MUSIC; i.e., the signal model vector at a correct incoming echo bearing from the sea is orthogonal to all of the noise eigenvectors. The algorithm finds the angle(s) at which this condition occurs. For example, with the SeaSonde, one does this first for a singleangle possibility where $\mathrm{N}-1=2$ eigenvectors are assumed to be noise, followed by the dualangle possibility where only $\mathrm{N}-2=1$ eigenvector remains as noise.

- Test to find which candidate bearings best-fit the data; Schmidt (1986) suggests the statistical chi-squared and F-tests. For the $\mathrm{N}=3$ SeaSonde system, which allows up to two angles for each radial speed from each Bragg peak, there can be from one to four angles found (each Bragg peak has independent information).

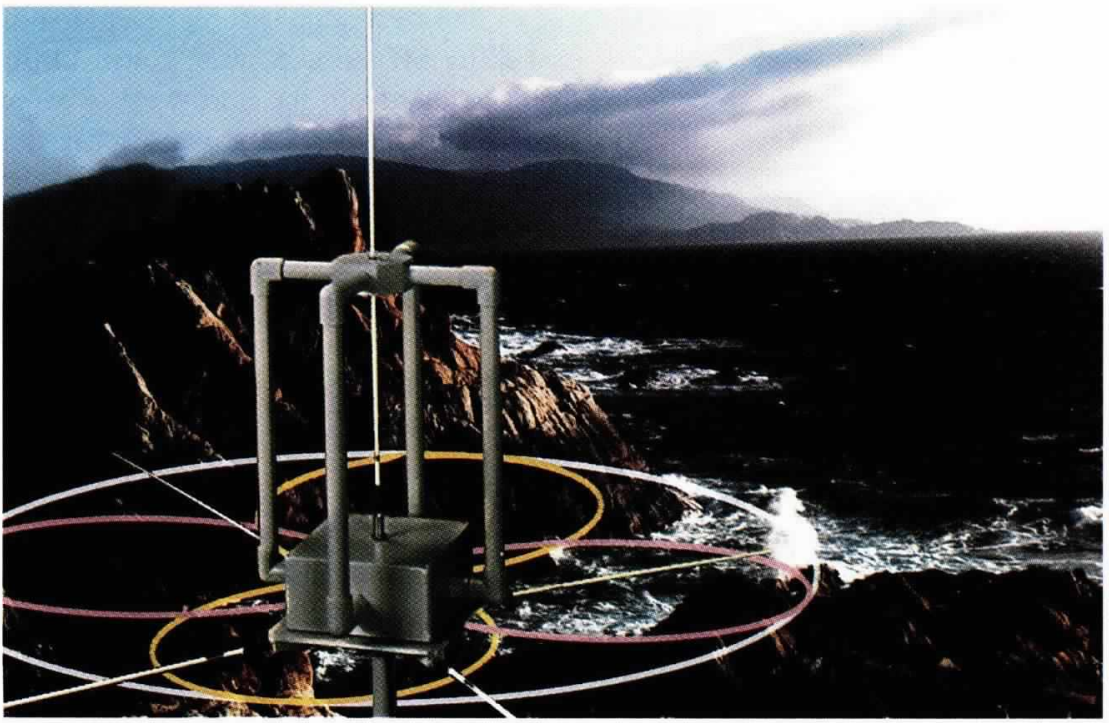

Fig. 1: Stylized view of a coastal SeaSonde crossed-loop/monopole receive antenna. Indicated at the antenna base are idealized patterns of the two loops (yellow and pink) and the vertical whip/monopole (white).

More antenna elements admit more bearing solutions, as well as better angular accuracy and resolving capability, as demonstrated below.

\section{Simulations Using the MUSIC Algorithm}

The most obvious scenarios for developing and optimizing bearing-estimation algorithms involve the collection of "ground-truthing" current measurements. However, such endeavors are expensive and generally woefully inadequate in terms of the amount of data collected. Sometimes overlooked is the capability of simulations to offer cost-effective ways to optimize candidate algorithms. In this case, one controls the input current pattern against which extracted estimates are compared on a point-by-point basis, giving statistical estimates of error. The essence of our simulations with the MUSIC algorithm are given in the following paragraph; details, such as the spectral models used to represent sea echo, can be found in Barrick and Lipa (1996).

We evaluated the performance of the MUSIC algorithm under different ocean wave and current conditions. In these cases, the following three deterministic patterns as a function of bearing angle were used as input to the simulations: 1) antenna element responses, 2) mean wind-wave (Bragg-signal) distribution, and 3) mean radial current speeds. In addition, the following two input variables were randomized: 1) the HF signal voltage at each bearing step, which was a zero-mean Gaussian sample whose variance followed the Bragg-signal distribution, and 2) radial current fluctuations about the mean current at each bearing step.

\section{Scenarios Studied}

In this paper we examine two ocean scenarios that are both commonly encountered and reason-

\section{Sometimes over-}

looked is the capabil-

ity of simulations to

offer cost-effective

ways to optimize

candidate algorithms. 
The first scenario

represents summertime

upwelling . . .

The second scenario

tested includes an

oceanographic front. ably complex. The first scenario represents summertime upwelling off the U.S. west coast. In this case, persistent winds from the northwest drive an offshore surface current to the southwest that brings deeper water to the surface along the coast. We consider the HF radar (SeaSonde) to be deployed on a straight coastline, as shown in the inset of Figure 2a. We assume that the wind-wave pattern has a typical $\cos ^{4}\left[\left(\varphi-\varphi_{\mathrm{w}}\right) / 2\right]$ distribution over bearing $\varphi$, where $\varphi_{\mathrm{w}}$ is the onshore wind direction (Fernandez et al., 1997). The mean radial current pattern for an annular range cell is modeled by the solid curve shown on Figure $2 \mathrm{a}$. This scenario is a good test for any algorithm because it has both single- and dual-valued speed ranges versus angle. The open circles on Figure $2 \mathrm{a}$ are estimates retrieved using MUSIC with no randomization of the input current, whereas the $x$ 's are the estimates for which a $\pm 10 \mathrm{~cm} / \mathrm{s}$ [root mean square (rms)] fluctuation was added to the mean pattern. These results are based on the optimized processing and algorithm parameters described in the next section.

The second scenario tested includes an oceanographic front. During this rare but interesting event, the current changes abruptly, as much as 50 $\mathrm{cm} / \mathrm{s}$, over a very short distance. We model this by shifting a portion of the upwelling current pattern (Fig. 2a) upward by $45 \mathrm{~cm} / \mathrm{s}$. The result (Fig. 2b) is quite stressing for any bearing estimation method, both because of the difficulty of resolving the discontinuity as well as the fact that the problem is now triple- or quadruple-valued. For example, a beam-scanning phased array with a 100-m antenna and resulting $14^{\circ}$ beamwidth would smear (i.e., low-pass-filter) through this front. To test the direction-finding MUSIC algorithm on this scenario, we added a $\pm 5 \mathrm{~cm} / \mathrm{s}$ (rms) random current component to the mean pattern. Results are presented in Figure $2 \mathrm{~b}$ for the standard, three-element antenna configuration used in the SeaSonde and for an expanded, five-element array with two additional monopole elements positioned as shown in the inset. The results are encouraging, even for this extreme frontal situation.

\section{Optimization of the MUSIC Algorithm}

Although continued experience always leads to improvements, a considerable amount of testing and comparisons with input have led to what we consider at this point to be the best mix of processing and algorithm parameters. These have been used to obtain the extracted points displayed in the above figures. They are based on two fairly stressing current scenarios with up to quadrupleangle solutions, as well as an abrupt jump. The optimum processing flow that we now recommend involves the following steps:

- Perform fast Fourier transforms on the $\mathrm{N}$ antenna signals every $256 \mathrm{~s}$. At $13 \mathrm{MHz}$ this results in a velocity resolution of $4.5 \mathrm{~cm} / \mathrm{s}$.
- Form three-sample covariance-matrix averages of these $\mathrm{N}$ signals versus Doppler shift (radial speed) with $\sim 30 \%$ overlap. This provides radial current estimates every $10 \mathrm{~min}$.

- Accumulate MUSIC-derived bearings versus radial velocity every 10 minutes over one hour.

- Apply a running average filter over $10^{\circ}$ in bearing angle stepped along every $2^{\circ}$.

To resolve the multiple-angle hypotheses within the MUSIC algorithm, the dual-angle solution is selected for our $\mathrm{N}=3$ SeaSonde if the following three conditions are all met: 1) The ratio of the largest two eigenvalues is $\ngtr 20,2$ ) the ratio of the two signal powers is $\ngtr 10$, and 3 ) the ratio of

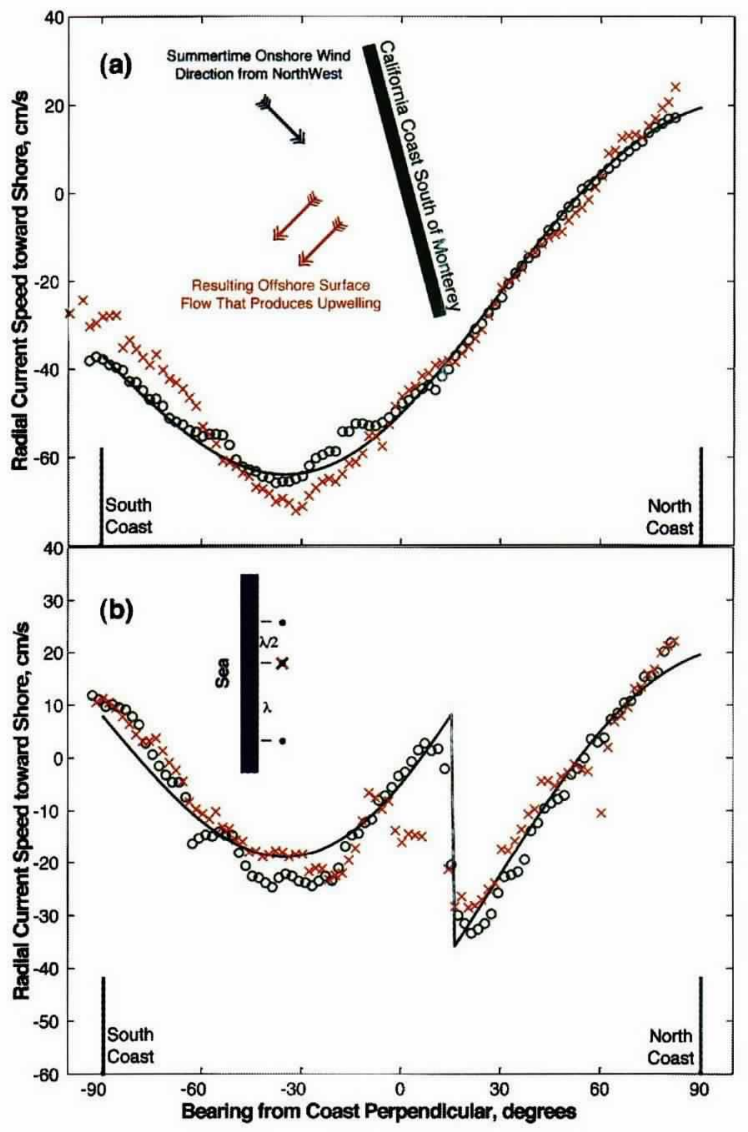

Fig. 2: Input mean radial current pattern versus bearing (solid curves) for California coastal upwelling scenario (a) showing retrieved MUSIC bearings for a three-element SeaSonde antenna with no random current component (green circles; rms error $=2.1 \mathrm{~cm} / \mathrm{s}$ ) and with a $10 \mathrm{~cm} / \mathrm{s} \mathrm{rms}$ random fluctuation (red crosses; $\mathrm{rms}=4.9 \mathrm{~cm} / \mathrm{s}$ ) and for a frontal jump of $45 \mathrm{~cm} / \mathrm{s}$ at $15^{\circ}$ bearing (b) showing retrieved bearings for a three-element SeaSonde antenna (red crosses; rms error $=5.7$ $\mathrm{cm} / \mathrm{s}$ ) and for a five-element antenna with two elements added off to the side (green circles; rms error $=3.8 \mathrm{~cm} / \mathrm{s})$. For case $(\boldsymbol{b})$ a random $5 \mathrm{~cm} / \mathrm{s}$ rms fluctuation was added to mean currents for both antenna configurations. 
the minor diagonal element product to the principal diagonal product of the $2 \times 2$ signal matrix is $1 / 3$, which is a measure of the matrix' positive definiteness.

The present SeaSonde operates with $\mathrm{N}=3$ antenna elements, all mounted on a single post. We examined various configurations where extra elements were added off to the side. The $\mathrm{N}=5$ configuration found to be best is that shown on Figure $2 \mathrm{~b}$ where whips (monopoles) are placed $\frac{1}{2}$ and 1 wavelength to either side of the crossed-loop/monopole unit.

\section{Summary and Conclusions}

Analysis of different ocean wind, wave, and current scenarios in simulations of radial current retrieval algorithms using HF radar backscatter should continue. This endeavor helps us both to develop new algorithms and to quantify the accuracy of these algorithms. The present study is a snapshot of some recent progress in this area applied to small-footprint, direction-finding systems. The same techniques can be applied to algorithms used with beam-forming systems (e.g., Barrick and Lipa, 1996). A summary of what we have found using the MUSIC algorithm reveals:

- Errors with the 3-antenna SeaSonde system using 1-h averaging are $\sim 2 \mathrm{~cm} / \mathrm{s}$ for dual-angle scenarios, like the upwelling example, when no random current fluctuations are added to the mean current pattern. These errors increase to $\sim 5 \mathrm{~cm} / \mathrm{s}$ when $\pm 10 \mathrm{~cm} / \mathrm{s} \mathrm{rms}$ fluctuations are added to the mean currents.

- Adding additional antenna elements to the basic three-element SeaSonde configuration improves its ability to map complicated current scenarios, such as the quadruple-angle front scenario. The five-element configuration tested here was able to retrieve the abrupt current jump across the front in the presence of $\pm 5 \mathrm{~cm} / \mathrm{s} \mathrm{rms}$ current noise. In the case of the smoother current patterns, such as the upwelling scenario, there is no appreciable accuracy difference between the three- and five-element antenna configurations.

\section{Acknowledgments}

We are grateful for the technical and editorial improvements made by Dr. Jeff Paduan of the Naval Postgraduate School.

\section{References}

Barrick, D.E., 1972: First-order theory and analysis of $\mathrm{MF} / \mathrm{HF} / \mathrm{VHF}$ scatter from the sea. IEEE Trans. Antennas Propag., AP-20, 2-10.

_- M.W. Evans and B.L. Weber, 1977: Ocean surface currents mapped by radar. Science, 198, 138-144.

_ and B.J. Lipa, 1986: Correcting for distorted antenna patterns in CODAR ocean surface measurements. IEEE J. Ocean. Engr., OE-11, 304-309.

- and B.J. Lipa, 1996: Comparison of direction-finding and beam-forming in HF radar ocean surface current mapping, Phase 1 SBIR Final Report. Contact No. 50DKNA-5-00092. National Oceanic and Atmospheric Administration, Rockville, MD.

Crombie, D.D., 1955: Doppler spectrum of sea echo at 13.56 $\mathrm{Mc} / \mathrm{s}$, Nature, 175, 681-682.

Fernandez. D.M.. H.C. Graber. J.D. Paduan and D.E. Barrick, 1997: Mapping wind direction with HF radar. Oceanography, 10, 93-95.

Gurgel, K.-W., 1997: Experience with shipborne measurements of surface current fields by radar. Oceanography, 10, 82-84.

Lipa, B.J. and D.E. Barrick, 1983: Least-squares methods for the extraction of surface currents from CODAR crossed-loop data: application at ARSLOE. IEEE $J$. Ocean. Engr., OE-8, 226-253.

Schmidt, R.O., 1986: Multiple emitter location and signal parameter estimation. IEEE Trans. Antennas Propag., AP34, 276-280.
. . a snapshot of

some recent progress

in this area applied

to small-footprint, direction-finding

systems. 